\title{
Análise do uso da terapia fotodinâmica no tratamento endodôntico com base em um Congresso Odontológico
}

\section{Analysis of the use of photodynamic therapy in endodontic treatment based on a Dental Congres}

Manuela Gouvêa Campêlo dos Santos*

Lívia Natália Sales Brito*

Lucas Emmanuell de Morais Neves*

Mariele Silva de Azevedo**

Thayana Karla Guerra dos Santos*

\section{Resumo}

Um dos objetivos do tratamento endodôntico é a máxima desinfecção do sistema de canais radiculares, bem como, a prevenção da sua reinfecção. A terapia fotodinâmica surge como um método coadjuvante na etapa de desinfecção obtendo significativa redução microbiana. Objetivo: traçar um panorama do perfil de uso da terapia fotodinâmica na endodontia no Brasil com base num Congresso de Odontologia e analisar o protocolo utilizado na desinfecção do sistema de canais radiculares. Materiais e método: realizou-se um estudo transversal, por meio da observação indireta dos resumos publicados nos anais da Sociedade Brasileira de Pesquisa Odontológica acerca do uso da terapia fotodinâmica na endodontia nos anos de 2013 a 2016. A coleta foi realizada por dois examinadores e as informações foram organizadas e analisadas no programa Statistical Program Software e apresentadas por meio da estatística descritiva. Resultados: foram encontrados 97 trabalhos com $37,1 \%$ no ano de $2016 ; 26,8 \%$ no ano de $2014 ; 18,6 \%$ em 2015 e 17,5\% em 2013. Observou-se que 40,2\% correspondiam a estudos in vitro, $68 \%$ tendo finalidade antibacteriana. Em 25,8\% associaram o hipoclorito de sódio com outras substâncias químicas auxiliares e 81,4\% utilizaram o hidróxido de cálcio como medicação intracanal. $O$ azul de metileno foi o fotossensibilizador mais descrito. Conclusão: a terapia fotodinâmica mostra-se promissora como coadjuvante ao tratamento endodôntico convencional. Fazem-se necessárias mais pesquisas, pois ainda não existe um protocolo padrão para o uso da terapia fotodinâmica na endodontia.

Palavras chaves: Endodontia; Lasers; Terapia Fotodinâmica.

\section{Introdução}

A infecção endodôntica ocorre com a proliferação de bactérias no interior dos canais radiculares que adentraram nesse meio através da exposição do dente ao ambiente oral, devido a lesões cariosas ou traumáticas, causando a formação de lesões perirradiculares persistentes ${ }^{1,2}$. Dessa forma, o tratamento endodôntico deve ser realizado como medida para eliminar e inativar os biofilmes bacterianos e seus subprodutos facilitando assim a reparação dos tecidos apicais ${ }^{1,3}$.

Durante o preparo químico mecânico utiliza-se instrumentos manuais ou mecanizados, concomitante a irrigação com substâncias químicas, e em alguns casos, o uso de medicação intra-canal entre as sessões clínicas, obtendo assim relativas taxas de sucesso na diminuição da quantidade de bactérias presentes nos canais radiculares ${ }^{2,4,5,6}$. Está bem evidenciado na atual literatura, que a desinfecção completa dos canais radiculares é bastante difícil, devido à diversidade dos sistemas de canais radiculares na região apical onde há maior predomínio de deltas apicais e formação de biofilme extraradicular, o que dificulta a eliminação de micro-organismos resistentes, tal como o Enterococcus Facaelis ${ }^{5,7,8}$.

O tratamento endodôntico convencional falha na inativação e eliminação das endotoxinas bacterianas como os lipopolissacarídeos que são liberados provocando infecções endodônticas graves que comprometem o sucesso da terapia endodôntica ${ }^{2,6}$.

\footnotetext{
MSc, Professora de Odontologia, Departamento de Odontologia da Universidade Estadual da Paraíba, Araruna, PB, Brasil.
}

Graduando em Odontologia pela Universidade Estadual da Paraíba. Araruna, PB, Brasil. 
O Enterococcus Faecalis é um anaeróbio facultativo que tem sido evidenciado em aproximadamente $77 \%$ dos casos, representando uma ameaça ao sucesso do tratamento ${ }^{3,9}$. Isso está associado a resistência contra os efeitos bactericidas dos agentes antimicrobianos mais utilizados como hipoclorito de sódio, clorexidina e hidróxido de cálcio ${ }^{2-3-4}$. Portanto, torna-se necessário novas formas de combater a infecção endodôntica ${ }^{4,5,10,11 \text {. }}$

A terapia fotodinâmica (TFD) é um método que utiliza um agente fotossensibilizador ativado por uma fonte de luz, através de um comprimento de onda específico, essa reação provoca a liberação de oxigênio reativo ou cria espécies reativas de oxigênio capazes de danificar a parede celular, proteínas da membrana celular e ácidos nucleicos das bactérias ${ }^{1,3,4,7,13}$. Os fossensibilizadores possuem um elevado grau de seletividade para atingir os micro-organismos sem provocar danos às células ${ }^{8}$.

Dessa forma, a TFD tem sido apontada como uma alternativa coadjuvante no tratamento endodôntico, pois apresenta potencial de aumentar a eficiência na descontaminação dos canais radiculares quando utilizada com a instrumentação convencional ${ }^{1,13}$. Além disso, tem efeito sobre a inativação de fatores de virulência liberados pelas bactérias gram-negativas e contra os lipopolissacarídeos ${ }^{6,13}$.

A publicação de artigos científicos em periódicos de reconhecido mérito e a apresentação de resumos em importantes eventos científicos é reconhecidamente uma parte importante no processo de intercâmbio de informações científicas. A Sociedade Brasileira de Pesquisa Odontológica ( $\mathrm{SBPqO}$ ) consiste no maior congresso de pesquisa científica em odontologia no Brasil e através deles pode-se avaliar o perfil dos estudos em diversas áreas da odontologia.

Sendo assim, através dos dados secundários dos resumos publicados nos anais da Sociedade Brasileira de Pesquisa Odontológica referente aos anos de 2013 a 2016 este estudo teve como objetivo de descrever o panorama de uso da terapia fotodinâmica na endodontia no Brasil, bem como, analisar sua eficácia e protocolos utilizados na desinfecção do sistema de canais radiculares.

\section{Materiais e método}

Esse trabalho tem caráter observacional, transversal e descritivo. Trata-se de uma pesquisa documental com base em dados secundários, onde foram analisados todos os trabalhos publicados nos anais da SBPqO referente aos anos de 2013 a 2016. A seleção dos trabalhos foi realizada por meio de dois pesquisadores previamente calibrados. Foram utilizados para busca os Descritores em Ciências da Saúde (DeCS) da Biblioteca Virtual em Saúde (BVS): Endodontia, Lasers, Terapia Fotodinâmica, além dos descritores Laserterapia e Ledterapia. Como critérios de inclusão, todas as pesquisas que abordassem a terapia fotodonâmica no tratamento endodontico, estudos in vitro e in vivo.

Após a seleção dos trabalhos, os pesquisadores buscaram informações sobre as seguintes variáveis: ano de publicação; tipo de estudo realizado; caracterização da pesquisa; indicação terapêutica; micro-organismos envolvidos; substância química utilizada; medicação intracanal utilizada; tipo de laser (Light Amplification by Stimulated Emission of Radiation); tipo do fotossensibilizador além dos protocolos para terapia fotodinâmica como comprimento de onda, potência, dose e tempo de irradiação.

Os dados obtidos foram dispostos em uma tabela informatizada criada em ambiente Excel Microsoft para facilitar a conversão em um banco de dados informatizados com o auxílio do Statistical Package for the Social Sciences (SPSS, v.20) para realizar a análise estatística das variáveis. A análise da distribuição dos dados foi realizada de forma descritiva expondo os resultados em porcentagens, frequências simples e relativas.

\section{Resultados}

Os resultados obtidos demonstraram que dos 97 trabalhos avaliados nos anais do SBPqo referente à terapia fotodinâmica, $37,1 \%(\mathrm{n}=36)$ foram publicados no ano de 2016; $26,8 \%(\mathrm{n}=26)$ no ano de 2014; $18,6 \%(n=18)$ em 2015 e por fim $17,5 \%(n=17)$ dos estudos foram publicados no ano de 2013. Demonstrando assim, certa prevalência de estudos que abordam esse tema durante os anos avaliados.

Com relação à caracterização do estudo, foi observada prevalência em pesquisa do tipo in vitro com dentes de humanos $40,2 \%$ ( $n=39$ ), seguida de in vitro não específico $30,9(\mathrm{n}=30)$. Os estudos em humanos corresponderam a $19,7 \%(n=22)$ com maior frequência para ensaios clínicos, e os que utilizaram para testes em camundongos laboratoriais totaliza$\operatorname{ram} 9,2 \%(\mathrm{n}=9)$.

Quanto à finalidade de uso da terapia fotodinâmica, 68\% ( $\mathrm{n}=66$ ) usaram como efeito antibacteriano, seguido de análise tecidual $4,1 \%(\mathrm{n}=4)$ e análise da fotobiomodulação 4,1\% ( $\mathrm{n}=4)$. Dos 97 estudos avaliados, 23,6\% ( $\mathrm{n}=23)$ não descreveram nos anais para que fins a terapia foi utilizada.

As pesquisas analisadas apresentaram, com relação aos micro-organismos envolvidos, predileção por Enterococcus Faecalis 38,1\% ( $\mathrm{n}=37)$, seguida por Candida Albicans em 6,2\% $(\mathrm{n}=6)$. Do total de estudos avaliados, 9,3\% (n=9) associaram Enterococcus Faecalis com outros micro-organismos, 2,1\% $(\mathrm{n}=2)$ associaram a Candida Albicans com outros e 2,1\% ( $\mathrm{n}=2$ ) associaram Enterococcus Faecalis com Candida Albicans. Em 42,3\% (n=41) dos trabalhos não mencionaram os micro-organismos envolvidos.

A substancia química auxiliar mais frequente, a medicação intracanal mais utilizada e o fotossensi- 
bilizador mais descrito nas pesquisas estão elencados respectivamente nas tabelas 1,2 e 3 .

$\mathrm{O}$ laser de baixa intensidade foi utilizado em $27,8 \%$ ( $n=27)$, já o de alta intensidade foi utilizado em 12,4\% (n=12) e o LED (Light Emitter Diode) utilizado em 15,5\% ( $\mathrm{n}=15)$. Em apenas um estudo foi utilizado o ultrassom associado ao laser e em $43,2 \%$ $(\mathrm{n}=42)$ não descreveram o laser utilizado.

O comprimento de onda mais prevalente estava na faixa entre 600 a $700 \mathrm{~nm}$ com uma porcentagem de 38,1 (n=37), acompanhada da faixa entre 400 a 500 em $9,3 \%(n=9)$ e $4,1 \%(n=4)$ entre 500 a $600 \mathrm{~nm}$. Comprimento de ondas maiores que $700 \mathrm{~nm}$ foram descritos em $2,1 \%(\mathrm{n}=2)$ e maiores que $800 \mathrm{~nm}$ em $3,1 \%(\mathrm{n}=3)$. De todos os estudos, $42(43,3 \%)$ não relataram o comprimento de onda utilizado.

Tabela 1 - Distribuição os trabalhos segundo a substância química auxiliar

\begin{tabular}{l|c|c}
\multicolumn{1}{c|}{ Substância } & Frequência (n) & $\%$ \\
\hline $\begin{array}{l}\text { Hipoclorito de Sódio isolada nas } \\
\text { diferentes porcentagens }\end{array}$ & 21 & 21,6 \\
$\begin{array}{l}\text { Hipoclorito de Sódio associado à outras } \\
\text { substâncias }\end{array}$ & 25 & 25,8 \\
Soro Fisiológico & 04 & 4,1 \\
Clorexidina & 08 & 8,2 \\
Não citado & 39 & 40,3 \\
\hline
\end{tabular}

Tabela 2 - Distribuição da pesquisa segundo a medicação intracanal

\begin{tabular}{l|c|r}
\hline \multicolumn{1}{c|}{ Medicação } & Frequência (n) & \multicolumn{1}{c}{$\%$} \\
\hline Hidróxido de Cálcio & 79 & $81,4 \%$ \\
Hidróxido de Cálcio associado a & 08 & $8,2 \%$ \\
outro medicamento & 09 & $9,3 \%$ \\
Paranomoclorofenol & 01 & 1,1 \\
Não citado & & \\
\hline
\end{tabular}

Tabela 3 - Distribuição dos trabalhos quanto ao fotossensibilizador

\begin{tabular}{l|c|c}
\multicolumn{1}{c|}{ Fotossensibilizador } & Frequência (n) & \multicolumn{1}{c}{$\%$} \\
\hline Azul de Metileno & 20 & $20,6 \%$ \\
Curcumina & 9 & $9,3 \%$ \\
Azul de Toluidina & 9 & $9,3 \%$ \\
Azul de Metileno associado com o & 4 & $4,1 \%$ \\
Azul de Toluidina & & \\
Não citado & 55 & 56,7 \\
\hline
\end{tabular}

O tempo estabelecido nos protocolos descritos variam de menos de um minuto a 30 minutos, apresentando a maior prevalência em menos de um minuto $11,3 \%(\mathrm{n}=11)$, acompanhada por dois minutos $10,3 \%(\mathrm{n}=10)$, quatro e cinco minutos ambos com $5,2 \%(\mathrm{n}=5)$. O número de estudos que não mencionam o tempo no protocolo foi de $(\mathrm{n}=71) 72,9 \%$.

\section{Discussão}

As pesquisas apresentadas durante as reuniões do SBPqO servem como referência para traçar o perfil da pesquisa odontológica desenvolvida no país ${ }^{14}$. Durante o congresso são apresentados trabalhos previamente selecionados, oriundos das diferentes regiões do Brasil e abrangendo todas as áreas do conhecimento em odontologia.

O tratamento endodôntico baseia-se na descontaminação eficaz do sistema de canais radiculares, como coadjuvante a terapia fotodinâmica entraria como potencializador do efeito antimicrobiano ${ }^{15}$. Embora não ocorra um consenso sobre um protocolo padrão para sua utilização na área da endodontia, algumas variáveis podem ser questionadas devido à falta de padronização: indicação terapêutica, micro-organismos envolvidos, substância química, medicação intracanal, tipo de laser, tipo de fotossensibilidador, concentração do fotossensibilizador, comprimento de onda, potência, dose e tempo de irradiação $0^{16}$.

Nesta investigação, a solução química irrigadora mais utilizada, correspondeu à solução de hipoclorito de sódio. Em seu estudo in vitro Neelakantab ${ }^{12}$ utilizaram o hipoclorito de sódio a 3\% e a curcumina fotoativada como solução irrigadora objetivando comparar a eficácia dessas duas substâncias e concluíram que a última apresentou significativamente maior capacidade antibacteriana. Outro estudo feito por Juric ${ }^{17}$ analisou o hipoclorito de sódio a $5,25 \%$ como irrigante e a solução QMiX, obtiveram como resultado que o hipoclorito de sódio foi capaz de reduzir 99,99\% Enterococcus faecalis sendo mais significante do que os outros protocolos utilizados.

Segundo Lopes ${ }^{18}$, o objetivo do uso das soluções irrigadoras durante o tratamento endodôntico é promover, além do efeito antimicrobiano, funções de lubrificação do instrumento endodontico, atividade solvente de tecido orgânico durante a etapa da instrumentação com a finalidade de eliminação ou máxima redução de microorganismos. A terapia fotodinâmica sozinha não teria tais efeitos, por isso seria uma etapa coadjuvante com finalidade de aumentar a redução microbriana ${ }^{15}$.

Em relação ao uso da medicação intracanal, a mais utilizada nos estudos encontrados foi a pasta de hidróxido de cálcio. Em concordância com Lopes ${ }^{18}$, a pasta quando utilizada como medicação intracanal, pode desempenhar atividades biológicas, químicas e físicas, obtendo efeito antimicrobiano, solubilizando matéria orgânica e atuando como barreira física. Em estudo in vitro, Miranda ${ }^{19}$ analisaram e compararam a terapia fotodinâmica, o uso adjuvante do EndoVac ${ }^{\circledR}$, um sistema de irrigação por pressão apical negativa, a terapia fotodinâmica em conjunto com o hidróxido de cálcio como medicação intracanal na redução dos níveis de Enterococcus faecalis. Os autores concluíram que o sistema EndoVac ${ }^{\circledR}$ e 
a TFD, em combinação ou não, foram tão eficazes quanto o desbridamento químico-mecânico associado com o hidróxido de cálcio na redução dos níveis de Enterococcus faecalis, ou seja, a TDP não foi superior aos outros métodos. Já no estudo de Asnaashar $^{20}$ avaliaram in vivo o efeito antimicrobiano da desinfecção fotoativada ao usar o azul de toluidina como fotossensibilizador e uma lâmpada LED após o tratamento convencional, comparando-o com a terapia com hidróxido de cálcio. Os autores concluíram que a TFD e a terapia com hidróxido de cálcio, como métodos auxiliares complementares à terapia de canal radicular, são eficientes na desinfecção do canal radicular. Contudo, quando comparou-se a terapia de hidróxido de cálcio à $\mathrm{TFD}$, esta alcançou uma maior redução do número de Enterococcus faecalis nos canais radiculares infectados.

Com relação aos fotossensibilizadores, conceituado como um corante que deve apresentar absorção ampla de acordo com o comprimento de onda da fonte luminosa, deve ter também estabilidade biológica, seletividade celular e baixo efeito tóxico. Os fotossensibilizadores mais utilizados para a TFD são o azul de metileno, seguido do azul de toluidina e curcumina. A dose utilizada para sucesso terapêutico é menor que a dose necessária para causar danos a células adjacentes ${ }^{21}$.

Com o avanço tecnológico dos lasers e suas aplicações na odontologia, notou-se uma crescente indicação terapêutica na qual utiliza esse instrumento. Isso se justifica por suas características, tais como a luz monocromática, coerência, facilidade da associação com o corante, entre outras. Existe uma diversificação de tipos de laser, porém o mais descrito na literatura para tal procedimento é o laser de diodos de baixa intensidade. No presente estudo foi verificado que o laser de baixa intensidade foi $o$ mais utilizado, corroborando com pesquisas recentes que sugerem o uso de lasers com comprimentos de onda entre 400-700 nm, sendo o comprimento de onda $660 \mathrm{~nm}$ mais utilizado e podendo inativar com sucesso taxas relativas de microrganismos ${ }^{1,13,21}$.

Em contrapartida, Susila ${ }^{2}$ avaliaram os efeitos da terapia fotodinâmica com irrigação na erradicação de patógenos comum na endodontia, utilizando o Laser de Diodo de alta potência a $1 \mathrm{~W}$ durante 30 segundos em modo pulsado como TFD, reduzindo com significância as colônias de Enterococcus faecalis e Streptococcus Mutans.

Para que a aplicação da TFD se estenda no interior de todo canal radicular é necessário utilizar um equipamento de diâmetro e formato adequado. Por esse motivo, para realizar a terapia na endodontia de forma satisfatória, a fibra óptica é essencial, a qual é adaptada a ponta do aparelho e é a responsável pela transmissão de energia em forma de luz do laser para o conduto. Possui características de flexibilidade e calibres compatíveis. Após entrar no canal, deve-se realizar movimentos helicoidais do ápice em direção a coroa ${ }^{21}$.
Outro fator observado em protocolos para TFD é o tempo de irradiação, o qual é avaliado como o tempo entre a aplicação do fotossensibilizador na área e a ativação da luz. Esse tempo é importante para que ocorra a difusão do corante e o contato com os microrganismos. De forma geral, o tempo relatado nos atuais protocolos se estende aproximadamente por 3 minutos ${ }^{15}$.

O desenvolvimento da pesquisa científica apresenta um importante papel para a geração de novos conhecimentos, novos protocolos e para o desenvolvimento do espírito crítico. É importante destacar uma limitação do presente trabalho que foi o fato de o estudo contemplar, exclusivamente, a análise dos resumos da $\mathrm{SBPqO}$, que apesar de constituir-se em um congresso de excelência na pesquisa, não é o único que pode representar o panorama nacional da pesquisa brasileira. Existem outros eventos científicos em diferentes áreas do conhecimento, que abordam tal temática. No entanto, a despeito dessa limitação, os resultados aqui descritos retratam o perfil da pesquisa científica a respeito da prática da terapia fotodinâmica.

Porém, verificou-se na presente pesquisa, que apesar de vários estudos tenham sido realizados, tanto in vitro como in vivo, obtendo resultados positivos para a utilização da TFD na endodontia, ainda não foram padronizados protocolos que estabeleçam parâmetros de relação entre a luz, tempo de exposição e fotossensibilizadores.

\section{Conclusão}

A terapia fotodinâmica mostra-se promissora como coadjuvante ao tratamento endodôntico convencional. Fazem-se necessárias mais pesquisas, com maior potencial de evidência científica, pois ainda não existe um protocolo padrão para o uso da terapia fotodinâmica na endodontia.

\section{Abstract}

One of the objectives of endodontic treatment is the maximum disinfection of the root canal system, as well as preventing its reinfection. Photodynamic therapy appears as a supporting method in the disinfection stage, obtaining significant microbial reduction. Objective: to outline a profile overview of the use of photodynamic therapy in endodontics in Brazil, based on a Dental Congress, and to analyze the protocol used in root canal system disinfection. Materials and method: a cross-sectional study was carried out by indirectly analyzing abstracts published in the proceedings of the Brazilian Society of Dental Research on the use of photodynamic therapy in endodontics, in the years from 2013 to 2016. Two examiners performed the collection; information were organized and analyzed in the Statistical Program Software and presented through descriptive statistics. Results: a total of 97 studies were found, of which $37.1 \%$ were published in 2016, 26.8\% in 2014, 
$18.6 \%$ in 2015 , and $17.5 \%$ in 2013. It was observed that $40.2 \%$ corresponded to in vitro studies, of which $68 \%$ were for antibacterial purpose. In $25.8 \%$ of the studies, there was an association of sodium hypochlorite with other auxiliary chemical substances and $81.4 \%$ used calcium hydroxide as intracanal medication. Methylene blue was the photosensitizer mostly described. Conclusion: photodynamic therapy is promising as a support to the conventional endodontic treatment. Further research is required, as there is no standard protocol for the use of photodynamic therapy in endodontics.

Keywords: Endodontics. Lasers. Photodynamic therapy.

\section{Referências}

1. Marinic K, Manoil D, Filieri A, Wataba JC. Schrenzel, J.; Lange, N.; Bouillaguet, S. Repeated exposures to blue light-activated eosin $\mathrm{Y}$ enhance inactivation of $\mathrm{E}$. faecalis biofilms, in vitro. Photodiagnosis and Photodynamic Therapy, 2015; 12: 393-400.

2. Susila AV, Sugumar R, Chandama CS, Subbarao CV. Combined effects of photodynamic therapy and irrigants in disinfection of root canals. Journal of Biophotonics, 2016; 9 (6): 603-609.

3. Tennert C, Drews AM, Walther V, Altenburguer MJ, Karygianni L, Wrbas KT, Hellwig E, Al-Ahmad A. Ultrasonic activation and chemicalmodification of photosensitizers enhancesthe effects of photodynamic therapy against $E n$ terococcus faecalis root-canal isolates. Photodiagnosis and Photodynamic Therapy, 2015; 12: 244-251.

4. Gomes-Filho JE, Sivieri-Araujo G, Sipert CR, Santos LMS, Queiroz IOA, Martins CM, Maia NKC, Cintra LTA, Dezan-Junior E, Bagnato VS, Chaves-Neto AH, Oliveira SHP. Evaluation of photodynamic therapy on fibroblast viability and cytokine production. Photodiagnosis and Photodynamic Therapy, 2016; 13: 97-100.

5. Juric IB, Plecko V, Pamduric DG, Anic I. The antimicrobial effectiveness of photodynamic therapy used as an additionto the conventional endodonticre-treatment: A clinical study. Photodiagnosis and Photodynamic Therapy, 2014; 11: 549555.

6. Shrestha A, Cordova M, Kishen A. Photoactivated Polycationic Bioactive Chitosan Nanoparticles Inactivate Bacterial Endotoxins. Journal of Endodontics, 2015; 41 (5): 686691.

7. Xhevdet A, Stubljar D, Kriznar I, Jukic T, Skvarc M, Veranic P, Alojz I. The Disinfecting Efficacy of Root Canals with aser Photodynamic Therapy. Journal of Lasers in Medical Sciences, 2014; 5 (1): 19-26.

8. Samiei M, Shahi S, Abdollahi AA, Eskandarinezhad M, Negahdari R, Pakseresht Z. The Antibacterial Efficacy of Photo-Activated Disinfection, Chlorhexidine and Sodium Hypochlorite in Infected Root Canals: An in Vitro Study. Iranian Endodontic Journal, 2016; 11 (13): 179-183.

9. Silva E, Coutinho-Filho WP, Andrade AO, Herrera DR, Coutinho-Filho TS, Krebs RL. Evaluation of Photodynamic Therapy Using a Diode Laser and Different Photosensitizers Against Enterococcus Faecallis. Acta Odontológica Latinoamericana, 2014; 27 (2): 63-65.

10. Costa LM, Matos FS, Correira AMO, Carvalho NC, Faria-e-Silva AL, Paranhos LR, Ribeiro MAG, Tooth color change caused by photosensitizers after photodynamic therapy: An in vitro study. Journal of Photochemistry and Photobiology B: Biology, 2016; 160: 225-228.
11. Shetestha A, Kishen A. Antibiofilm Efficacy of Photosensitizer-functionalized Bioactive Nanoparticles on Multispecies Biofilm. Journal of Endodontics, 2014; 40 (10): 604-1610.

12. Neelakantab P, Cheng QC, Ravichandran V, Mao T, Sriraman P, Sridharan S, Subbarao C, Sharma S, Kichen A. Photoactivation of curcumin and sodiumhypochlorite to enhance antibiofilmefficacy in root canal dentin. Photodiagnosis and Photodynamic Therapy, 2015; 12: 108-114.

13. Garcez AS, Arantes-Neto JG, Sellera DP, Fregnani ER. Effects of antimicrobial photodynamictherapy and surgical endodontic treatmenton the bacterial load reduction andperiapical lesion healing. Three yearsfollow up. Photodiagnosis and Photodynamic Therapy, 2015; 12: 575-580.

14. Cormack EF, Silva Filho CF. A pesquisa científica odontológica no Brasil. Rev Assoc Paul Cir Dent. 2000; 54: 242-7.

15. Amaral RR, Sa DM, Menezes AJAC. Terapia fotodinamica antimicrobiana na Endodontia: revisão de literatura. Pós em Revista, 2015; 2015 (2): 39-41.

16. Oliveira MS, Rodrigues SFD, Cintra LTA, Gomes-Filho JE Jacinto RC, Sivieri-Araujo G. O emprego da terapia fotodinâmica como coadjuvante à infecção endodôntica. Archives of health investigation, 2016; 5: 119.

17. Juric IB.; Plecko V, Anic I, Plesko S, Jakovljevic S, Rocca JP, Medioni E. Antimicrobial efficacy of photodynamic therapy, Nd:YAG laser and QMiX solution against Enterococcus faecalis biofilm. Photodiagnosis and Photodynamic Therapy, 2016; 13 (1): 238-243

18. Lopes HP, Siqueira Jr JF. Endodontia: biologia e técnica. 3 ed. Rio de Janeiro: Gen/Guanabara Koogan, 2010. 851p.

19. Miranda RG, Santos EB, Souto RM, Gusman H, Colombo APV. Ex vivo antimicrobial efficacy of the EndoVac ${ }^{\circledast}$ system plus photodynamic therapy associated with calcium hydroxide against intracanal Enterococcus faecalis. International Endodontic Journal, 2012; 46 (6): 499-505.

20. Asnaashari M, Ashraf H, Rafmati A, Amini N. A comparison between effect of photodynamic therapy by LED and calcium hydroxide therapy for root canal disinfection against Enterococcus faecalis: A randomized controlled trial. Photodiagnosis and Photodynamic Therapy, 2017; 17 (1): 226-232.

21. Clovis MB, Alexandre SB, Roberto BG, Eduardo KD. Terapia fotodinámica en el tratamiento endodontico y quirúrgico. Rev. odontol. Dominic, 2016; 12: 30-34.

Endereço para correspondência:

Manuela Gouvêa Campêlo dos Santos

Av. Cel. Pedro Targino, s/n - Centro

58233-000 Araruna - PB, Brasil

E-mail: manuelagouvea@hotmail.com

Recebido: 04 / 12 /2016. Aceito: 02 /03/2017. 